\title{
ACHADOS DOS POTENCIAIS EVOCADOS SOMATOSSENSITIVOS E MOTORES NA MIELOPATIA ASSOCIADA AO HTLV-I
}

\author{
Daniela Oliveira de Andrade ${ }^{1}$
}

\begin{abstract}
RESUMO - Foram estudados 63 pacientes com diagnóstico de paraparesia espástica tropical/ mielopatia associada ao HTLV-I (PET/ MAH) através de potencial evocado somatossensitivo (PESS) e 42 destes pacientes através de potencial evocado motor (PEM). Todos os pacientes apresentaram história clínica típica de MAH e sorologia para HTLV-I confirmada por Western blot. Dos pacientes estudados por PESS 51/63 (81\%) alterados em membros inferiores e $11(17,5 \%)$ deles estavam também em membros superiores. Dos pacientes estudados por PEM 37/42 estavam alterados, 34/42 (81\%) em membros inferiores e 25/42 (59,5\%) em membros superiores. Um alto percentual da amostra apresentou alterações das vias corticoespinhais nos quatro membros ao PEM, e ausência de alterações em membros superiores ao PESS, evidenciando a correlação entre o tempo de condução motora central para membros superiores e a gravidade clínica da PET/ MAH $(p<0,01)$. Não foi evidenciado correlação entre o tempo de historia clínica e os achados dos PESS e PEM $(p=0,69)$.
\end{abstract}

PALAVRAS-CHAVE: HTLV-I, paraparesia espástica tropical, potenciais somatosensoriais evocados, potencial evocado motor.

\section{Somatosensitive and motor evoked potentials in HTLV-I associated mylopathy}

\begin{abstract}
This study investigated 63 patients with HTLV-I associated myelopathy / tropical spastic paraparesis (HAM/ TSP) by somatosensitive evoked potentials (SEPS) and 42 of them by motor evoked potentials (MEP). All the patients had typical clinical history of HAM, serum samples tested positive for antibodies to HTLVI screened for Particle Aglutination, ELISA and complemented by Westem blot test. In patients studied by SEPs of lower limbs 51/63 (81\%) were abnormal and 11 of them (17.5\%) were abnormal in upper limbs also. In patients studied by MEP $37 / 42$ were abnormal, 34/42 (81\%) in lower limbs and 25/42 (59.5\%) in upper limbs. A high percent of the population studied had abnormalities of the corticospinal tracts on the four limbs at the PEM, without abnormalities in upper limbs by SEPs, showing the correlation between central motor conduction time in upper limbs and the clinical severity of HAM/TSP $(p<0.01)$. It was not found correlation between time of disease and the results of the SEPs and MEP $(p=0.69)$.
\end{abstract}

KEY WORDS: HTLV-I, tropical spastic paraparesis, somatosensitive evoked potential, motor evoked potential.

A apresentação clínica da paraparesia espástica tropical/mielopatia associada ao HTLV-I (PET/MAH) é caracterizada por paraparesia espástica associada a alterações sensitivas, disfunção vesical, impotência e obstipação intestinal, além de outras patologias como leucemia-linfoma', artralgias, alveolite linfocítica e uveíte ${ }^{2}$. A maior prevalência de HTLVI em doadores de sangue $(1,35 \%)^{3}$ em nosso país é a da capital do Estado da Bahia, Salvador. A prevalência de portadores de HTLV-I avaliada através de estudo de campo na cidade de Salvador foi $1,76 \%{ }^{4}$.

Alguns estudos evidenciaram o envolvimento das vias somatossensitivas posteriores através do potencial evocado somatossensitivo (PESS) em $44 \%^{5}, 85,5 \%{ }^{6}$ e $86,3 \%{ }^{7}$ dos pacientes com PET/
MAH. Estudo das vias motoneuronais por estimulação magnética transcraniana em pacientes com PET/MAH evidenciou um percentual de alterações de $17,6 \%$ para membros superiores e $73,8 \%$ em membros inferiores ${ }^{8}$. A análise das vias somatossensitivas posteriores por PESS neste estudo não evidenciou exames alterados em membros superiores e um percentual de alterações de $31,3 \%$ em mem$b$ ros inferiores, percentual inferior ao descrito na literatura ${ }^{5-7}$. Os achados do potencial envocado motor (PEM $)^{8}$ evidenciaram um percentual de exames alterados em membros superiores significantemente maior que o percentual de exames alterados em membros superiores no PESS. Esses autores sugerem que em pacientes com PET/MAH há comprometimento mais importante das vias cortico-

Rede Sarah de Hospitais da Reabilitação: 'Médica Neurofisiologista Clínica do Hospital Sarah, Salvador BA, Brasil.

Recebido 27 Setembro 2004, recebido na forma final 19 Janeiro 2005. Aceito 29 Março 2005. 
espinhais descendentes que das vias ascendentes da coluna posterior. Shimizu et al. ${ }^{9}$ evidenciaram correlação entre o tempo de condução motora central (TCMC) para membros superiores e a gravidade clínica da PET/ MAH, não havendo correlação entre o TCMC para membros inferiores e a gravidade clínica da PET/MAH. Essa correlação não foi evidenciada por Suga et al. ${ }^{8}$, que encontraram percentual de exames alterados em membros superiores significantemente maior que em membros inferiores, ao PEM $(p<0,01)$.

Este estudo visa descrever e comparar os achados do estudo combinado de PESS e PEM nos quatro membros em pacientes com PET/ MAH.

\section{MÉTODO}

Foram investigados 119 pacientes com PET/MAH através de PESS e/ou PEM de janeiro de 2000 a março de 2004. As características dos pacientes quanto a sexo, idade atual, idade do início da doença, tempo de duração da doença e resultados dos exames sorológicos, neurofisiológicos e de neuroimagem foram levantados retrospectivamente dos registros em prontuários eletrônicos.

Foram considerados como critérios de inclusão a presença de anticorpos no soro e/ou líquor, detectados através de dois métodos de triagem (aglutinação de part ículas e ELISA) e confirmados por Western blot, investigados no laboratório deste hospital, e associação com história clínica de mielopatia lentamente progressiva.

Os pacientes que apresentaram neuropatia periférica por outra etiologia, compressão medular, comprome timento do sistema nervoso central (SNC) de etiologia vascular, degenerativa ou infecciosa como HIV, ou história de cirurgia prévia da coluna ou encéfalo foram excluídos do estudo. Todos os pacientes analisados foram investigados por ressonância magnética (RM) da coluna cervico-torácica neste hospital.

Um total de 63 pacientes foi incluído na nossa análise dos dados do PESS e 42 pacientes quanto aos achados do PEM e PESS. A exclusão dos pacientes deveu-se a presença de osteoart rose da coluna, neuropatia periférica diabética e ausência de investigação por RM da coluna cervico-torácica.

Os exames de PESS foram realizados com os pacientes em posição supina usando um aparelho Keypoint. O PESS foi realizado por estímulo elétrico percutâneo no nervo mediano, bilateralmente nos membros superiores (MMSS) e tibial posterior bilateralmente nos membros inferiores (MMII) na intensidade suficiente para p roduzir leve contração muscular involuntária deflagrados por estimulador de superfície com duração de 0,2 ms e freqüência de $1 \mathrm{~Hz}$ no total de 500 estímulos em cada segmento.

PESS do nervo mediano foi obtido após captação com eletrodos de superfície (disco $5 \mathrm{~mm}$ ) na região supra-clavicular (ponto de Erb), processo espinhoso cervical C7 e no escalpe em C3' e C4' (sistema internacional 10-20) em montagem referencial Erb contralateral e Fz respectiva mente. O PESS do nervo tibial foi obtido após captação na região lombar L1-L2, cervical C7 e no escalpe em Cz. Eletrodos de referência colocados na crista ilíaca e Fz. Impedância mantida abaixo de $5 \Omega$. Filtros de 50-1000 $\mathrm{Hz}$. Os componentes examinados foram latências absolutas e interpicos de N9, N13 e N19 nos MMSS e latências absolutas e interpicos de PL, N30 e N/P37 nos MMII.

As vias motoneuronais foram estudadas utilizando o PEM Magpro (Dantec). Durante o procedimento o paciente foi colocado em posição supina e relaxado. Pulsos magnéticos liberados através de bobina circular MC125 de diâmetro externo de $120 \mathrm{~mm}$, gerando um campo máximo de 2,2 Tesla no centro da bobina. A intensidade do estímulo foi $80 \%$ da máxima de saída para captação em membros superiores e $100 \%$ da máxima de saída para captação em membros inferiores, emitindo pulsos bifásicos e amplificados com filtros de $20 \mathrm{~Hz}$ e $3 \mathrm{kHz}$, freqüência máxima de $1 \mathrm{~Hz}$ e duração de 0,2 ms. Foram realizados estímulos corticais com bobina posicionada no vértex para captação nos MMSS e C3'e C4' contralaterais à captação nos (sistema internacional 10-20). Durante os estímulos os pacientes realizaram leve contração do músculo estudado com intuito de facilitar a resposta. O potencial de ação muscular composto (PAMC) foi captado por eletrodos de superfície posicionados sobre o ventre muscular (ponto motor) dos músculos extensor curto dos dedos (EDB) nos MMII e abdutor do dedo mínimo (ADM) nos MMSS. Quatro estímulos subseqüentes e seus potenciais foram registrados de cada sítio de estimulação. O tempo de condução motora central TCMC foi calculado da subtração da menor latência cortical, da latência periférica (TCMC=LC-LP). A latência periférica foi calculada através da fórmula $M+F-1 / 2$, onde $M$ corresponde a latência da onda $M$ e $F$ a latência da onda $F$.

O PESS e o PEM foram considerados alterados quando o tempo de condução em um dos quatro membros estivesse prolongado ou resposta ausente. Para análise do PEM levou-se em consideração a razão das amplitudes dos potenciais corticais e a onda M (PEM/M). A análise considerou como valores normais a média \pm 2 desvio padrão (DP) dos achados dos $\mathrm{PESS}^{10}$ e $\mathrm{PEM}^{11}$ descritos na literatura e similar aos valores encontrados em estudo de normatização de grupo controle de 103 pacientes realizado neste hospital ${ }^{12}$.

Para análise estatística dos dados foi utilizado o teste Qui-quadrado de McNemar e Pearson, t-Student para diferença entre duas proporções e médias, utilizando a significância de 5\%.

\section{RESULTADOS}

Dos pacientes estudados $68,3 \%$ eram mulheres (43/63). A faixa etária no início dos sintomas variou de 6 a 75 anos (média 40,5 anos, DP 15,8 anos) e no momento do estudo de 15 a 86 anos (média 


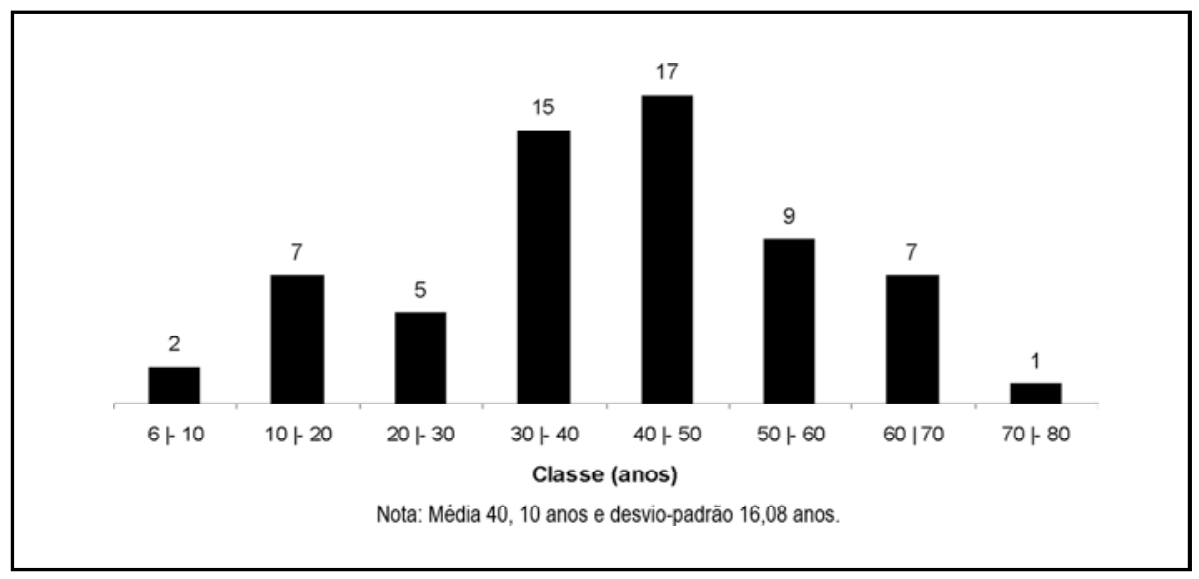

Fig 1. Distribuição dos pacientes portadores de PETIMAH segundo a idade no início da doença.

49,4 anos, DP 15,5 anos). Apenas nove pacientes iniciaram os sintomas com idade inferior a 20 anos (Fig 1).

Dos pacientes investigados $57(90,5 \%)$ apresentavam paraparesia espástica e $5(8 \%)$, tetraparesia. Apenas um paciente iniciou os sintomas com artralgia e urgência miccional há quatro anos, ainda não apresentando déficit de força em MMII.

Foram avaliados 63 pacientes por PESS, sendo $12(19 \%)$ exames normais e $51(81 \%)$ alterados. Nenhum paciente apresentou achados alterados apenas em membros superiores, $40(78,4 \%)$ apresentaram alterações em membros inferiores e 11 $(21,6 \%)$ em três ou quatro membros (Tabela 1$)$.

Foram avaliados 42 pacientes por PEM e PESS (Tabelas 2 e 3). Neste grupo, $5(11,9 \%)$ tiveram PEM normais e $37(88,1 \%)$ alterados. Não houve acometimento isolado nos MMSS, $12(32,4 \%)$ pacientes apresentaram alterações apenas em MMII e $25(67,6 \%)$ em três ou quatro membros. Os PESS realizados neste grupo foram normais em 9/42 $(21,4 \%)$ pacientes e alterados em $33(78,6 \%)$. Não houve acometimento isolado nos MMSS, 26 (78,8\%) pacientes mostraram alterações em MMII e 7 $(21,2 \%)$ em três ou quatro membros.

Aplicou-se o teste de McNemar em 32/37 pacientes, que correspondem aos pacientes que apresentavam alterações ao PESS e ao PEM. Excluiu-se desta análise 4 pacientes por apresentarem normalidade em um dos exames. Este teste avaliou a gravidade da doença segundo o PEM e PESS dividindo os pacientes em dois grupos, aqueles com alterações apenas em MMII e aqueles com alterações em três a quatro membros. Dentre os pacientes identificados por comprometimento apenas dos MMII não houve discordância entre os dois exames, enquanto dentre os pacientes com com-
Tabela 1. Frequência de exames alterados em MMSS e MMII dentre os 51/63 pacientes avaliados por PESS.

\begin{tabular}{ccc}
\hline Resultado & $\mathrm{n}$ & $\%$ \\
\hline $1 \mathrm{MS}$ & 0 & 0 \\
$2 \mathrm{MS}$ & 0 & 0 \\
3 ou 4 membros & 11 & 21,6 \\
$1 \mathrm{MI}$ & 4 & 7,8 \\
$2 \mathrm{MI}$ & 36 & 70,6 \\
Total & 51 & 100 \\
\hline
\end{tabular}

MS, membro superior; MI, membro inferior.

Tabela 2. Frequência de exames alterados ao PESS em MMSS e MMII dentre os 33/42 pacientes avaliados por PESS e PEM.

\begin{tabular}{ccc}
\hline Resultado do PESS & $\mathrm{n}$ & $\%$ \\
\hline 1MS & 0 & 0 \\
2MS & 0 & 0 \\
3 ou 4 membros & 7 & 21,2 \\
1MI & 4 & 12,1 \\
2MI & 22 & 66,7 \\
Total & 33 & 100 \\
\hline
\end{tabular}

$\mathrm{MI}$, membro inferior; MS, membro superior.

Tabela 3. Frequência de exames alterados ao PEM em MMSS e MMII dentre os $37 / 42$ pacientes avaliados por PESS e PEM.

\begin{tabular}{ccc}
\hline Resultado do PEM & $\mathrm{n}$ & $\%$ \\
\hline 1MS & 0 & 0 \\
2MS & 0 & 0 \\
3 ou 4 membros & 25 & 67,6 \\
1MI & 1 & 2,7 \\
2MI & 11 & 29,7 \\
Total & 37 & 100 \\
\hline
\end{tabular}

MS, membro superior; MI, membro inferior. 
Tabela 4. Exames alterados ao PEM e PESS dentre os 32/42 pa cientes que apresentaram alterações mútuas nestes dois testes.

\begin{tabular}{lcccc}
\hline & & PESS & 3 ou 4 & \\
& & MMII & membros & \\
\hline \multirow{2}{*}{ PEM } & MMII & 10 & 0 & 10 \\
& 3 ou 4 membros & 14 & 8 & 22 \\
\multirow{2}{*}{ Total } & & 24 & 8 & 32 \\
\hline
\end{tabular}

MMII, alterações apenas em membros inferiores; 3 ou 4 membros, alterações em membros inferiores e em um dos membros superiores.

prometimento de três a quatro membros ocorre u discordância, que foi estatisticamente significante $(p<0,01)$. O PEM identificou 14/32 pacientes com comprometimento de três a quatro membros, enquanto estes mesmos pacientes apresentaram comprometimento apenas de MMII pelo PESS (Tabela 4).

O tempo de história da doença nesta amostra variou de 1 a 25 anos (média 10,5 anos e DP 6,0 anos). Os pacientes foram divididos em dois grupos, aqueles que apresentaram PEM alterados apenas em MMII e aqueles que apresentaram PEM alterados em 3 ou 4 membros. O grupo de pacientes com alterações apenas em MMIl evidenciou a média do tempo de doença de 10,2 anos, DP de 8,0 anos e o grupo com alterações em 3 ou 4 memb ros, média de 10,7 anos, DP de 5,0 anos. Utilizando o teste t-Student, não houve diferença significativa entre tempos de doença dos grupos $(p=$ $0,69)$. Verificou-se através do teste t-Student que não houve diferença quanto a idade $(p=0,614)$, e através do teste Qui-quadrado de Pearson que a distribuição quanto ao sexo foi homogênea ( $p=$ 0,844 ) entre os dois grupos.

\section{DISCUSSÃO}

O estudo das vias motoneuronais e somatossensitivas posteriores por PEM e PESS no mesmo grupo de pacientes não mostrou diferença significativa $(p=0,219)$ do percentual de alterações, $88,1 \%$ e $78,6 \%$ respectivamente. Apenas três estudos realizaram análise combinada do PESS e PEM em um pequeno número de pacientes com $\mathrm{PET} / \mathrm{MAH}^{8,9,13}$.

A gravidade da mielopatia em nosso grupo variou desde um quadro de lombalgia associado à incontinência urinária a tetraparesia. $O$ percentual de pacientes com achados de disfunção ao PEM, no grupo com alterações em MMII, foi $65 \%$ (24/37) e no grupo com alterações em 3 ou 4 membros foi $35,1 \%$ (13/37), evidenciando diferença estatisticamente significante $(p=0,011)$. O PESS evidenciou $21,2 \%$ (7/33) dos pacientes com alterações em 3 a
4 membros e um percentual equivalente a $78,8 \%$ (26/33) dos pacientes com alterações limitadas aos MMII, evidenciando uma diferença estatisticamente significante $(p<0,01)$.

Esses achados no mesmo grupo de pacientes evidenciaram um comprometimento mais severo das vias corticoespinhais em relação aos fascículos grácil e cuneiforme, sugerindo o início das alterações em MMII evoluindo para os MMSS. Isto está de acordo com os achados dos exames de neuroimagem que evidenciam comprometimento mais frequente da medula torácica e atrofia difusa da medula em casos mais graves ${ }^{14}$.

Não foi encontrada diferença estatisticamente significante entre o tempo de história da doença e os resultados do PEM, de modo similar aos achados de Suga et al. ${ }^{8}$. Acreditamos que esta análise pode ter sido prejudicada pela existência de diferenças entre as duas amostras que as tornam heterogêneas, como as vias de transmissão da doença se sanguínea, sexual ou longitudinal, ou outros fatores de erro.

\section{REFERÊNCIAS}

1. Freitas V, Gomes I, Bittencourt A, et al. Adult T-cell leukemia-lymphoma in a patient with HTLV-I/II associated myelopathy. Arq Neuropsiquiatr 1997, 55:325-328.

2. Melo A, Gomes I, Mattos I. Mielopatias por HTLV-I na cidade de Salvador, Bahia. Arq Neuropsiquiatr 1994,52:443-444.

3. Galvão-Castro B, Loures L, Proietti F, et al. Distribution of human Tcell lymphotropic virus type I among blood donors: a nation-wide Brazilian study. Transfusion (Paris) 1997;37:242-243.

4. Dourado I, Alcântara LCJ, Barreto ML, et al. HTLV-I in general population of Salvador, Brazil, a city with African ethnic and sociodemographic characteristics. J Acquir Immune Defic Syndr 2003;34:527-531.

5. Moritoyo M, Arimura K, Arimura Y, et al. Study of lower limb somatosensory evoked potentials in 96 cases of HTLV-I-associated myelopathy/tropical spastic paraparesis. J Neurol Sci 1996;138:78-81.

6. Cruz MW, Corrêa RB, Puccioni-Sohler M, et al. Eletroneuromiografia e potenciais evocados somatossensitivos na mielopatia pelo HTLV-I. Arq Neuropsiquiatr 1998;56:756-762.

7. Castillo JL, Cartier L, Araya F, et al. Evoked potential abnormalities in progressive spastic paraparesis associated to HTLV-I. Acta Neurol Scand 1991;83:151-154.

8. Suga R, Tobimatsu S, Kira J, et al. Motor and somatosensory evoked potentials findings in HTLV-I associated myelopathy. J Neurol Sci 1999;167:102-106.

9. Shimizu H, Shiga Y, Fujihara K, et al. Clinical and physiological significance of anormally prolonged central motor conduction time in HAM/ TSP. J Neurol Sci 2001, 185:39-42.

10. Chiappa KH. Short-latency somatosensory evoked potentials: methodology. In Chiappa KH (ed.). Evoked potentials in clinical medicine, $2^{\text {nd }}$ ed. New York: Raven Press, 1989:307-370.

11. Dvorák J, Herdmann J, Vohánka S. Motor evoked potentials by means of magnetic stimulation in disorders of the spine. Meth Clin Neurophysiol 1992, 3:45-64.

12. Fonte Boa PMO. Potencial evocado Motor por estimulação magnética transcraniana e radicular: estudo normativo para os músculos abdutor digiti minini e extensor digitorum brevis. Dissertação (Mestrado) Faculdade de Medicina de Ribeirão Preto, Universidade de São Paulo. Ribeirão Preto,1997.

13. Ugawa $Y$, Kohara N, Shimpo T, Mannen T. Central motor and sensory conduction in adrenoleukomyeloneuropathy, cerebrotendinous xanthomatosis HTLV-I associated myelopathy and tabes dorsalis. J Neurol Neurosurg Psychiatry 1988;51:1069-1074.

14. Sasaki S, Komori T, Maruyama S, Takeishi M, Iwasaki Y. An autopsy case of human T lymphotropic virus type I-associated myelopathy (HAM) with a duration of 28 years. Acta Neuropathol 1990;81:219-222. 